\title{
THREE-DIMENSIONAL THERMAL BUCKLING ANALYSIS OF FUNCTIONALLY GRADED CYLINDRICAL PANELS USING DIFFERENTIAL QUADRATURE METHOD (DQM)
}

\author{
Seyed A. Ahmadi, Hadi Pourshahsavari \\ Babol University of Technology, Department of Mechanical Engineering, Babol, Iran \\ e-mail: ali_ahmadi1366@yahoo.com
}

\begin{abstract}
Thermal buckling analysis of functionally graded cylindrical panels subjected to various conditions is discussed in this paper. Buckling governing equations are solved using the differential quadrature method. It is assumed that the mechanical properties of the panel are graded through thickness according to a power function of the thickness variable. The panel is assumed to be under the action of three types of thermal loading including uniform temperature rise and variable temperature rise in the axial and radial direction. In the present study, the effects of power law index, panel angle, different thermal load conditions and geometric parameters on the buckling behavior of functionally graded curved panels are studied. The results obtained through the present method are compared to the finite element solutions and the reported results in the literature. A desirable compatibility is concluded.
\end{abstract}

Keywords: thermal buckling, curved panel, functionally graded material, differential quadrature method

\section{Introduction}

Due to special mechanical properties, circular cylindrical panels are widely used in engineering structures such as pressure vessels, nuclear reactors, spacecrafts and jet engine exhausts. Due to the increasing demands for heat-resisting, energy absorbing, light-weight elements and high structural performance requirements in extremely high temperature environments and highspeed industries such as fusion reactors, aircraft and aerospace structures the use of special materials with high thermal and mechanical resistance has gained much popularity by many researchers. The applications of functionally graded materials (FGMs) have attracted much attention in the past two decades since they were first reported by Koizumi (1993). FGMs are composite materials, microscopically inhomogeneous, in which mechanical properties vary smoothly and continuously from one surface to the other. The main advantage of FGMs is that the ceramic component provides high temperature resistance due to its low thermal conductivity while the metal component prevents fracture induced by thermal stresses due to the hightemperature gradient in a very short period of time. When these are subjected to a thermal loading, the determination of thermal buckling capacity of these structures is important to achieve an optimized design in cost and weight.

Buckling analyses of various structures were carried out by many researchers. A review of research on the buckling response of plates and shells in a temperature environment was presented by Thornton (1993). He did some research on thermal buckling of plates and shells. In his work, he described elastic thermal buckling of metallic as well as composite plates and shells. Murphy and Ferreira (2001) investigated thermal buckling analysis of imperfect flat plates based on the energy consideration. They showed the ratio of the critical temperature for a perfect rectangular plate to that of an imperfect plate as a function of the initial imperfection amplitude. Mahayni (1966) studied thermal buckling behavior of doubly curved isotropic panels using Galerkin's 
method. Chang and Chui (1991) carried out bifurcation buckling analysis of composites under the action of uniform temperature change using higher order transverse shear deformation theory and the finite element method. Earlier, the Differential Quadrature Method introduced by Jang et al. (1989), was applied only to rectangular plates and lately it was considered for shells. Mirfakhraei and Redekop (1998) used the Differential Quadrature Method to study buckling behavior of circular cylindrical shells. Alibeigloo and Kani (2010) and Haftchenari et al. (2007) used this method to study cylindrical shells as well.

The study of structures of functionally graded materials has received considerable attention in recent years. Buckling of functionally graded plates under thermal loads was studied by Javaheri and Eslami (2002b). They used classical plate theory and obtained nonlinear equilibrium and linear stability equations using variational formulations. Shahsiah and Eslami (2003) considered effects of various temperature distributions on thermal buckling of simply supported FG cylindrical shells, using the first order shear deformation theory, however the temperature dependency of material properties was not included. Thermoelastic stability of FG cylindrical shells subjected to various thermal load conditions was studied by $\mathrm{Wu}$ et al. (2005). Thermal buckling analysis of functionally graded plates considering simply supported boundary conditions by using the first shear deformation theory was carried out by Wu (2004). He reached the stability equation of functionally graded shells using Donnell's shell theory and presented its closed-form solution. Buckling analysis of FG plates using a higher order theory was presented by Javaheri and Eslami (2002a). It was shown that higher order shear deformation theory accurately predicts the buckling behavior, whereas the classical plate theory overestimates the critical loads. Breivik (1997) discussed the buckling response of composite cylindrical panels under the action of mechanical and thermal loading. Zhao et al. (2007) and Zhao and Liew (2010) used the element-free kp-Ritz method for thermal and mechanical buckling analysis of functionally graded cylindrical shells. They obtained three-dimensional buckling equations of the shell based on the Donnell shell theory and presented a closed form solution to predict buckling loads caused by thermal loads and critical edge displacement in the longitudinal direction.

In this paper, buckling analysis of cylindrical panels made of a functionally graded material subjected to three types of thermal loading is investigated. To obtain the buckling load of the cylindrical panels, the Differential Quadrature Method (DQM) is used to discretize differential equations obtained based on the second Piola-Kirchhoff stress tensor using three-dimensional theory of elasticity by Akbari Alashti and Ahmadi (2014). The material properties are assumed to be temperature independent and vary continuously along the thickness according to a power law function while Poisson's ratio of the material is taken to be constant. Effects of various parameters including panel curvature, grading index, various thermal load conditions and geometric ratios on the buckling behavior of the curved panels are investigated. Numerical results are validated against finite element calculations and results that are available in the offered literature.

\section{Governing equation for buckling}

Consider a thick cylindrical panel made of ceramic and metallic materials with the inner radius $R_{1}$, mid-surface radius $a$, thickness $h$ and length $L$. The geometric parameters and the cylindrical coordinate system. i.e. $r, \theta$ and $x$-coordinates are shown in Fig. 1.

The components of the displacement field in this coordinate system are expressed as $w, v$ and $u$, respectively. Assume that the material is isotropic, inhomogeneous with Young's modulus varying continuously in the thickness direction, i.e. from ceramic in the inner layer to metallic in the outer layer according to the following formula

$$
V_{m}=\left(\frac{2 z+h}{2 h}\right)^{K} \quad V_{c}+V_{m}=1
$$




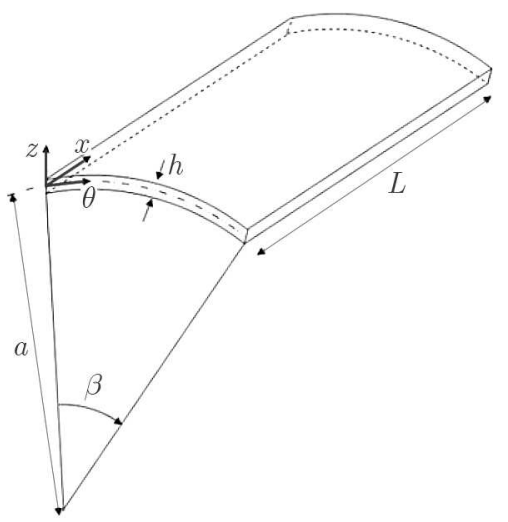

Fig. 1. Geometry of a cylindrical panel

where $V_{c}$ and $V_{m}$ represent the volume fractions of the ceramic and metallic constituent and $K$ denotes the volume fraction index that indicates the material variation profile through the FG shell thickness. Thus, the Young modulus in the radial direction is assumed to vary according to the power law in the following forms

$$
E(z)=E_{c}+E_{m c}\left(\frac{2 z+h}{2 h}\right)^{K} \quad E_{m c}=E_{m}-E_{c}
$$

where $E_{m}$ and $E_{c}$ denote the elastic modulus of the metal and ceramic, respectively. The material composition varies smoothly from the outer surface $(z=h / 2)$ of the shell as metal to the inner surface $(z=-h / 2)$ as ceramic. Material properties of the shell are assumed to be independent of the temperature field and Poisson's ratio is considered to be constant throughout the thickness of the shell.

In order to calculate buckling loads of panels, the buckling equations obtained by Akbari Alashti and Ahmadi (2014) are used.

In this work, also the finite element linear or bifurcation buckling analysis of the cylindrical panel using ANSYS suite of program is carried out. The eigen buckling analysis predicts theoretical buckling strength of a shell made of a linear elastic material. This analysis is used to predict the bifurcation point on an $F-U$ diagram using a linearized model of the elastic structure. It is a technique used to determine buckling pressures at which the structure becomes unstable and their corresponding buckling mode shapes. The basic form of the eigen buckling analysis is

$$
\mathbf{K} \phi=\lambda_{i} \mathbf{S} \phi
$$

where $\mathbf{K}, \phi_{i}, \lambda_{i}$ and $\mathbf{S}$ are the structural stiffness matrix, eigenvector, eigenvalues and stress stiffness matrix, respectively.

Eight noded quadrilateral shell elements, namely Shell281, are used to model the thick cylindrical shell. The elements can handle membrane, bending and transverse shear effects and are able to form the curvilinear surface satisfactorily. The elements are suitable for modeling of the layer and have the stress stiffening, large deflection and large strain capabilities.

Boundary conditions of shell panels are defined using equilibrium equations. For the initial and perturbed equilibrium positions, we have

$$
\begin{aligned}
& \sigma_{r r}\left(a+\frac{h}{2}, \theta\right)=\sigma_{r r}\left(a-\frac{h}{2}, \theta\right)=0 \quad \tau_{r \theta}\left(a+\frac{h}{2}, \theta\right)=\tau_{r \theta}\left(a-\frac{h}{2}, \theta\right)=0 \\
& \tau_{r x}\left(a+\frac{h}{2}, \theta\right)=\tau_{r x}\left(a-\frac{h}{2}, \theta\right)=0
\end{aligned}
$$


Boundary conditions at the panel edges are defined as:

— up and down edge, $x=0, L$

Simply supported: $\quad w=v=\sigma_{x x}^{\prime}=0$

Clamped: $\quad w=v=u=0$

— lateral edges, $\theta=0, \beta$

Simply supported: $\quad w=\sigma_{\theta \theta}^{\prime}=u=0$

Clamped: $\quad w=v=u=0$

\section{Calculation of buckling load}

In this work, two types of panels are considered:

Case 1. The panel is assumed to be simply supported at lateral edges and clamped at two ends. Therefore, thermal variation causes no axial stress on the panel, $N_{\theta}=0$.

Case 2. We assume that the panel has clamped boundary conditions at all edges. For this case thermal loading cause axial and circumferential stresses at the panel walls, $N_{x} \neq 0$, $N_{\theta} \neq 0$.

By substituting the components of the displacement field in the stress-strain and linear straindisplacement equations and the resulted expression in the buckling equations, the equilibrium equations are defined in terms of components of the displacement field.

In the present work, a polynomial expansion based on the Differential Quadrature Method applied by Bellman and Casti (1971) is used to discretize and solve the obtained buckling equations. According to this method, the first order derivative of the function $f(x)$ can be approximated as a linear sum of all functional values in the domain

$$
\left.\frac{d f}{d x}\right|_{x=x_{i}}=\sum_{j=1}^{N} w_{i j}^{(1)} f\left(x_{j}\right) \quad \text { for } i=1,2, \ldots, N
$$

where $w_{i j}^{(1)}$ is the weighting coefficient and $N$ denotes the number of grid points $x_{i}$ in the domain. There are different methods for calculation of the weighting coefficients matrix, see Shu (2000). Here, the weighting coefficients of the first order derivatives are defined based on the Lagrange interpolation polynomials as

$$
w_{i j}^{(1)}=\frac{M^{(1)}\left(x_{i}\right)}{\left(x_{i}-x_{j}\right) M^{(1)}\left(x_{j}\right)} \quad \text { for } \quad i \neq j \quad w_{i i}^{(1)}=\frac{M^{(2)}\left(x_{i}\right)}{2 M^{(1)}\left(x_{j}\right)}
$$

where

$$
\begin{aligned}
& M^{(1)}\left(x_{i}\right)=\prod_{\substack{k=1 \\
k \neq i, j}}^{N}\left(x_{i}-x_{k}\right) \quad N\left(x_{i}, x_{j}\right)=M^{(1)}\left(x_{i}\right) \delta_{i j} \\
& M^{(2)}(x)=N^{(2)}\left(x, x_{k}\right)\left(x-x_{k}\right)+2 N^{(1)}\left(x, x_{k}\right)
\end{aligned}
$$

and for higher order derivatives, we have

$$
\begin{aligned}
& w_{i j}^{(r)}=r\left(w_{i j}^{(1)} w_{i i}^{(r-1)}-\frac{w_{i j}^{(r-1)}}{x_{i}-x_{j}}\right) \quad \text { for } i, j=1,2, \ldots, N \quad r=2,3, \ldots, N-1 \\
& w^{(r)} i i=-\sum_{j=1, j \neq i}^{N} w^{(r)} i j
\end{aligned}
$$


Now, applying the above formulation to the buckling equations, we have

$$
\begin{aligned}
\mathcal{G}_{2} \sum_{l=1}^{N} a_{i, l}^{(2)} w_{l, j, k}+\frac{\mathcal{G}_{2}}{r} \sum_{l=1}^{N} a_{i, l}^{(1)} w_{l, j, k}+G(z) \sum_{n=1}^{Q} b_{j, n}^{(2)} w_{i, n, k}+\mathcal{G}_{1} \sum_{l=1}^{N} \sum_{n=1}^{Q} a_{i, l}^{(1)} b_{j, n}^{(1)} u_{l, n, k} \\
\quad-\frac{\mathcal{G}_{2}}{r^{2}} w_{i, j, k}+\frac{\mathcal{G}_{1}}{r} \sum_{l=1}^{N} \sum_{m=1}^{M} a_{i, l}^{(1)} c_{k, m}^{(1)} v_{l, j, m}+\frac{G(z)}{r^{2}} \sum_{m=1}^{M} c_{k, m}^{(2)} w_{i, j, m}+\frac{\mathcal{G}_{3}}{r^{2}} \sum_{m=1}^{M} c_{k, m}^{(1)} v_{i, j, m} \\
+\sigma_{x}^{0} \sum_{n=1}^{Q} b_{j, n}^{(2)} w_{i, n, k}+\sigma_{\theta \theta}^{0} \frac{2}{r^{2}} \sum_{m=1}^{M} c_{k, m}^{(1)} v_{i, j, m}+\sigma_{\theta \theta}^{0} \frac{1}{r^{2}} B_{i, j, k}-\sigma_{\theta \theta}^{0} \frac{1}{r^{2}} \sum_{m=1}^{M} c_{k, m}^{(2)} w_{i, j, m}=0 \\
G(z) \sum_{l=1}^{N} a_{i, l}^{(2)} v_{l, j, k}+\frac{\mathcal{G}_{1}}{r} \sum_{l=1}^{N} \sum_{m=1}^{M} c_{k, m}^{(1)} a_{i, l}^{(1)} w_{l, j, m}+\frac{\mathcal{G}_{3}}{r^{2}} \sum_{m=1}^{M} c_{k, m}^{(1)} w_{i, j, m}+G(z) \sum_{n=1}^{Q} b_{j, n}^{(2)} v_{i, n, k} \\
\quad+\frac{G(z)}{r} \sum_{l=1}^{N} a_{i, l}^{(1)} v_{l, j, k}-\frac{G(z)}{r^{2}} v_{i, j, k}+\frac{\mathcal{G}_{2}}{r^{2}} \sum_{m=1}^{M} c_{k, m}^{(2)} v_{i, j, m}+\frac{\mathcal{G}_{1}}{r} \sum_{n=1}^{Q} \sum_{m=1}^{M} c_{k, m}^{(1)} b_{j, n}^{(1)} u_{i, n, m} \\
\quad+\sigma_{x}^{0} \sum_{n=1}^{Q} b_{j, n}^{(2)} v_{i, n, k}-\sigma_{\theta \theta}^{0} \frac{2}{r^{2}} \sum_{m=1}^{M} c_{k, m}^{(1)} w_{i, j, m}+\sigma_{\theta \theta}^{0} \frac{1}{r^{2}} v_{i, j, k}-\sigma_{\theta \theta}^{0} \frac{1}{r^{2}} \sum_{m=1}^{M} c_{k, m}^{(2)} v_{i, j, m}=0 \\
G(z) \sum_{l=1}^{N} a_{i, l}^{(2)} u_{l, j, k}+\mathcal{G}_{2} \sum_{n=1}^{Q} b_{j, n}^{(2)} v_{i, n, k}+\mathcal{G}_{1} \sum_{l=1}^{N} \sum_{n=1}^{Q} b_{j, n}^{(1)} a_{i, l}^{(1)} w_{l, n, k}+\frac{G(z)}{r} \sum_{l=1}^{N} a_{i, l}^{(1)} u_{l, j, k} \\
\quad+\frac{G(z)}{r^{2}} \sum_{m=1}^{M} c_{k, m}^{(2)} u_{i, j, m}+\frac{\mathcal{G}_{1}}{r} \sum_{m=1}^{M} c_{k, m}^{(1)} v_{i, j, m}+\frac{\mathcal{G}_{1}}{r} \sum_{n=1}^{Q} b_{j, n}^{(1)} w_{i, n, k} \\
\quad+\sigma_{x}^{0} \sum_{n=1}^{Q} b_{j, n}^{(2)} u_{i, n, k}-\sigma_{\theta \theta}^{0} \frac{1}{r^{2}} \sum_{m=1}^{M} c_{k, m}^{(2)} u_{i, j, m}=0 \\
\\
\quad
\end{aligned}
$$

where

$$
\mathcal{G}_{1}=G(z)+\lambda(z) \quad \mathcal{G}_{2}=2 G(z)+\lambda(z) \quad \mathcal{G}_{3}=3 G(z)+\lambda(z)
$$

and $a_{i j}^{(k)}, b_{i j}^{(k)}$ and $c_{i j}^{(k)}$ denote the weighting coefficients of the $k$-th order derivative in the $r, \theta$ and $x$-direction, respectively; $N, Q$ and $M$ are grid point numbers in the $r, \theta$ and $x$-direction, respectively. The critical value of the buckling load is obtained by solving the set of equations presented in the matrix form as

$$
\left[\begin{array}{ll}
\mathbf{B}_{B} & \mathbf{B}_{D} \\
\mathbf{D}_{B} & \mathbf{D}_{D}
\end{array}\right]\left[\begin{array}{c}
d_{b} \\
u \\
v \\
w
\end{array}\right]=\sigma\left[\begin{array}{cc}
0 & 0 \\
\mathbf{D}_{B G} & \mathbf{D}_{D G}
\end{array}\right]
$$

where the sub-matrices $\mathbf{B}_{B}, \mathbf{B}_{D}$ and $\mathbf{D}_{B G}, \mathbf{D}_{D}, \mathbf{D}_{B}, \mathbf{D}_{D G}$ are found from the boundary conditions and governing equations, respectively. Equation (3.6) is transformed into the standard eigenvalue equation, as

$$
\left(-\mathbf{D}_{B G} \mathbf{B}_{B}^{-1} \mathbf{B}_{D}+\mathbf{D}_{D G}\right)^{-1}\left(-\mathbf{D}_{B} \mathbf{B}_{B}^{-1} \mathbf{B}_{D}+\mathbf{D}_{D}\right)\left[\begin{array}{lll}
u & v & w
\end{array}\right]^{\mathrm{T}}-\sigma \mathbf{I}\left[\begin{array}{lll}
u & v & w
\end{array}\right]^{\mathrm{T}}=0
$$

from which, the eigenvalues of $\sigma$ can be found. The smallest value of $\sigma$ is found to be the buckling load. 


\section{Thermal loading}

\subsection{Uniform temperature rise}

The temperature changes uniformly through the thickness and remains constant in the longitudinal and circumferential directions of the panel. This thermal variations induces only normal stress, and the parameter $\Phi$ is defined as

$$
\sigma=\frac{N}{h} \quad N=-\frac{\Phi}{1-\nu}
$$

and

$$
\begin{aligned}
& \Phi=\int_{-\frac{h}{2}}^{\frac{h}{2}}\left[E_{m}+E_{c m}\left(\frac{2 z+h}{2 h}\right)^{K}\right]\left[\alpha_{m}+\alpha_{c m}\left(\frac{2 z+h}{2 h}\right)^{K}\right] \Delta T(x, \theta, z) d z \\
& \Rightarrow \quad \Phi=\left(E_{c} \alpha_{c} h+\frac{\left[E_{c}\left(\alpha_{m}-\alpha_{c}\right)+\alpha_{c}\left(E_{m}-E_{c}\right)\right] h}{K+1}+\frac{\left(\alpha_{m}-\alpha_{c}\right)\left(E_{m}-E_{c}\right) h}{2 K+1}\right) \Delta T_{c r}
\end{aligned}
$$

Substituting buckling stress obtained by numerical solution into Eq. (4.1) and (4.2), helps us to obtain the thermal buckling load $\Delta T_{c r}$.

\subsection{Non-uniform temperature rise in the axial direction}

In this case, the assumed temperature varies in the longitudinal direction according to the following formula

$$
T=\Delta T\left(\frac{x}{L}\right)^{n}+T_{m} \quad \Delta T=T_{c}-T_{m} \quad n>0
$$

where $T_{m}$ is the temperature at the metal surface of the panels. According to the above equations, axial stresses caused by the temperature rise have the same variation in this direction. The critical stresses are obtained by considering the effects of this loading in the discretized governing equations and then, the buckling temperatures are achieved using equations (4.1) and (4.2).

\subsection{Non-uniform temperature rise in the radial direction}

The functionally graded materials are designed in order to resist against high temperature rise by ceramic, so the temperature change will be quite different at the two sides of FGM structures. The temperature distribution across the thickness is a function of the $z$ coordinate as follows

$$
T=\Delta T\left(-\frac{z}{h}+\frac{1}{2}\right)^{q}+T_{m} \quad-\frac{h}{2}<z<\frac{h}{2} \quad \Delta T=T_{c}-T_{m}
$$

The parameter $\Phi$ is defined as

$$
\Phi=\int_{-\frac{h}{2}}^{\frac{h}{2}}\left[E_{m}+E_{c m}\left(\frac{2 z+h}{2 h}\right)^{K}\right]\left[\alpha_{m}+\alpha_{c m}\left(\frac{2 z+h}{2 h}\right)^{K}\right]\left[\Delta T\left(-\frac{z}{h}+\frac{1}{2}\right)^{q}+T_{m}\right] d z
$$

The buckling temperature rise will be obtained using equation (4.1). For example, for $q=1$, the parameter $\Phi$ is given as

$$
\begin{aligned}
\Phi= & \left(E_{c} \alpha_{c} h+\frac{\left[E_{c}\left(\alpha_{m}-\alpha_{c}\right)+\alpha_{c}\left(E_{m}-E_{c}\right)\right] h}{K+1}+\frac{\left(\alpha_{m}-\alpha_{c}\right)\left(E_{m}-E_{c}\right) h}{2 K+1}\right) T_{m} \\
& +\frac{h}{(K+1)(K+2)(2 K+1)}\left\{E_{c} \alpha_{c} K^{3}+\frac{7}{2} E_{c} \alpha_{c} K^{2}\right. \\
& \left.+\alpha_{c} K\left[2\left(\frac{E_{m}-E_{c}}{4}+E_{c}\right)\left(\alpha_{m}-\alpha_{c}\right)+\left(2\left(E_{m}-E_{c}\right)+\frac{7}{2} E_{m}\right)\right]+E_{m} \alpha_{m}\right\} \Delta T_{c r}
\end{aligned}
$$




\section{Numerical results and discussion}

In order to illustrate the results of the presented method for an inhomogeneous shell, a functionally graded cylindrical shell made of aluminum and alumina is considered. Young's modulus is assumed to be temperature independent and vary smoothly in the radial direction according to a power law distribution of the volume fraction of the constituent materials. Young's modulus for alumina at the inner surface and for aluminum at the outer surface is assumed to be $E_{c}=380 \mathrm{GPa}$ and $E_{m}=70 \mathrm{GPa}$, respectively. It is also assumed that Possion's ratios of the constituent materials are constant and equal to 0.3. At the first step, the buckling temperatures calculated by the present study are validated against the results reported in the literature.

Figures 2 and 3 plot the critical temperature changes of the complete shell with $L=a=1$ against the ratio of the thickness to mid surface radius of the shell $h / a$ for the uniform temperature rise loading. The results are compared to the finite element results and those reported by Breivik (1997).
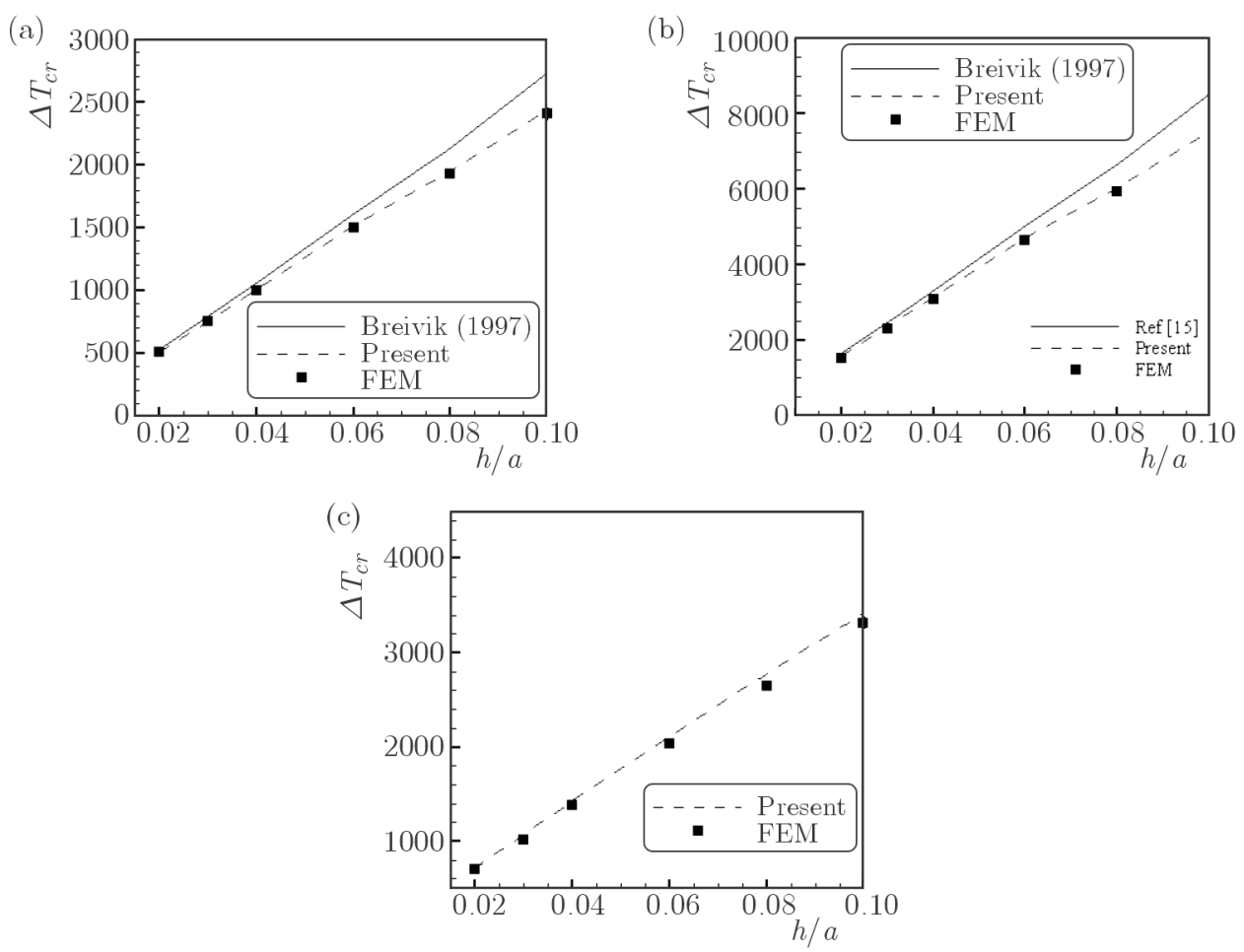

Fig. 2. Comparisons of the critical temperature of the complete shell under uniform temperature rise, (a) aluminum, (b) alumina, (c) functionally graded shell $K=1$

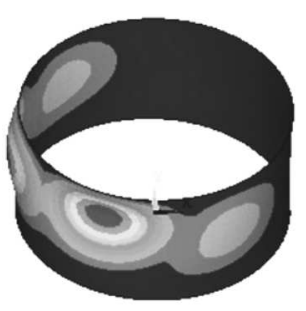

$h / a=0.1$

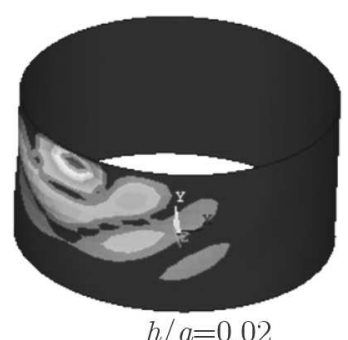

$h / a=0.02$

Fig. 3. Buckling mode shapes of complete shells made of aluminum under uniform temperature rise

It is evident that the results of the presented method are in good agreement with the finite element results and those of Breivik (1997). It can be seen from these figures that the critical 
buckling temperature increases linearly as the ratio of $h / a$ increases, and also the difference between the results increases when the relative thickness grows. It is because of the fact that Breivik (1997) used Donnell's theory to obtain the buckling equation of the thin shell. This equation creates an overestimation in the prediction of buckling load for a thick shell. The effects of panel angle on the buckling temperature are shown in Fig. 4. The results are compared to the results reported by $\mathrm{Wu}$ et al. (2005). The results are obtained for the panel (case 1) under uniform and non-uniform temperature rise in the radial direction, and the panel is assumed to be made of aluminum with $a=1 \mathrm{~m}, L / a=1$ and $h / a=0.02$.

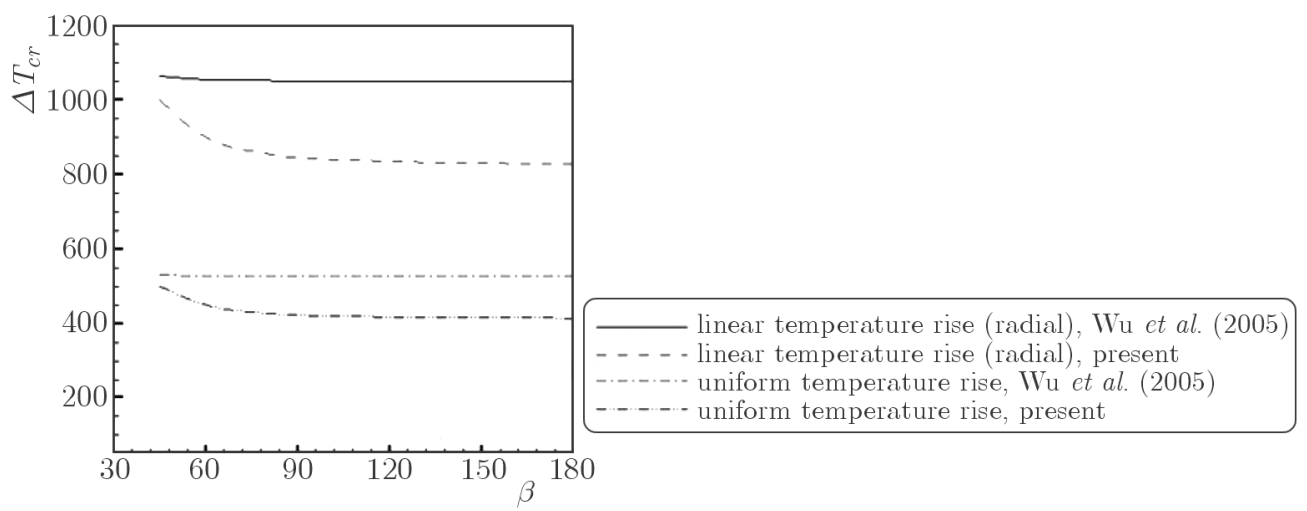

Fig. 4. Comparisons of the critical temperature of the homogeneous panel (case 1) made of aluminum with different angles $\left(T_{m}=0\right)$

(a)

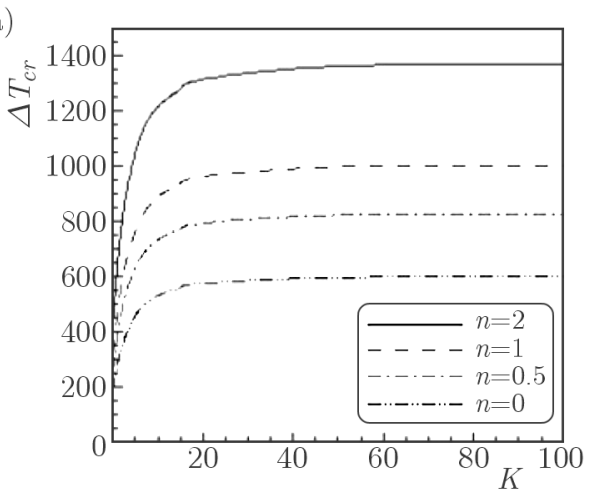

(b)

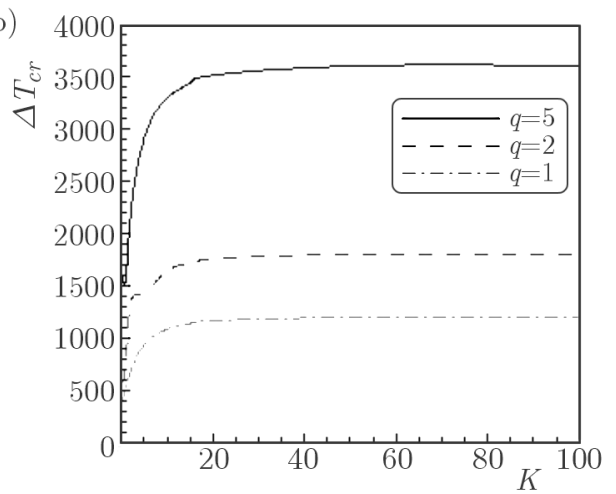

(c)

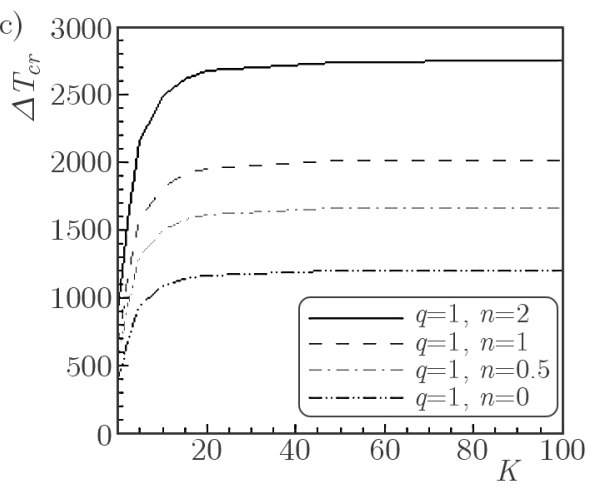

Fig. 5. Effect of the volume fraction index $K$ on the buckling temperature of the panel (case 1), non-uniform temperature rise in: (a) axial direction, (b) radial direction, (c) combined loading $\left(T_{m}=0\right)$

To make calculations following $\mathrm{Wu}$ et al. (2005), the critical stress is obtained first through the given formula and then substituted into Eq. (4.6). It can be inferred from Fig. 4 that the buckling temperature changes decrease when the panel angel increases, and for higher angles the 
results approach constant values. The results obtained based on Donnell's theory and obtained by $\mathrm{Wu}$ et al. (2005), show low variation of the buckling load versus panel angles.

Next, variation of the critical buckling temperature for the panel (case 1) with $L=a=1 \mathrm{~m}$, $h=0.01 \mathrm{~m}$ and $\beta=1 \mathrm{rad}$ under non-uniform temperature loading versus material gradient index $K$, are presented.

It is obvious from Fig. 5 that as the material gradient index $K$ increases from 1 to 10 , the critical buckling temperature grows rapidly and, for higher values, the results approach constant values. It is also evident that the critical buckling temperatures increase as the value of $K$ increases. The main reason for such an increase is the fact that a higher value of $K$ corresponds to a ceramic-richer panel, which usually has a higher thermal strength than a metal-richer one. Figure 6 shows the buckling temperature versus the ratio of $h / a$ for the panel (case 1) with $L=1 \mathrm{~m}$ and $\beta=1 \mathrm{rad}$ for three types of loading, i.e. uniform temperature rise and non-uniform temperature rise in the axial and radial direction. Buckling modes obtained through the finite element program for the uniform temperature rise are illustrated in Fig. 7.

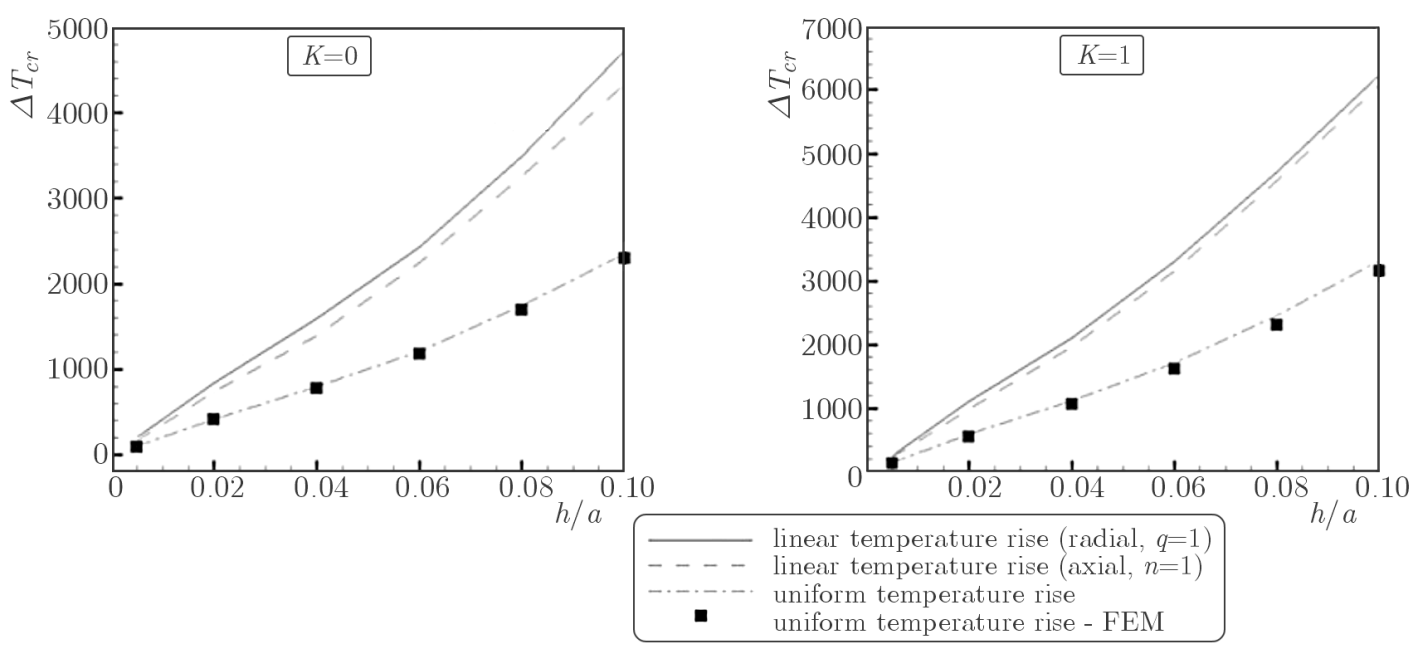

Fig. 6. Buckling temperature of the panel (case 1) versus $h / a\left(T_{m}=0\right)$
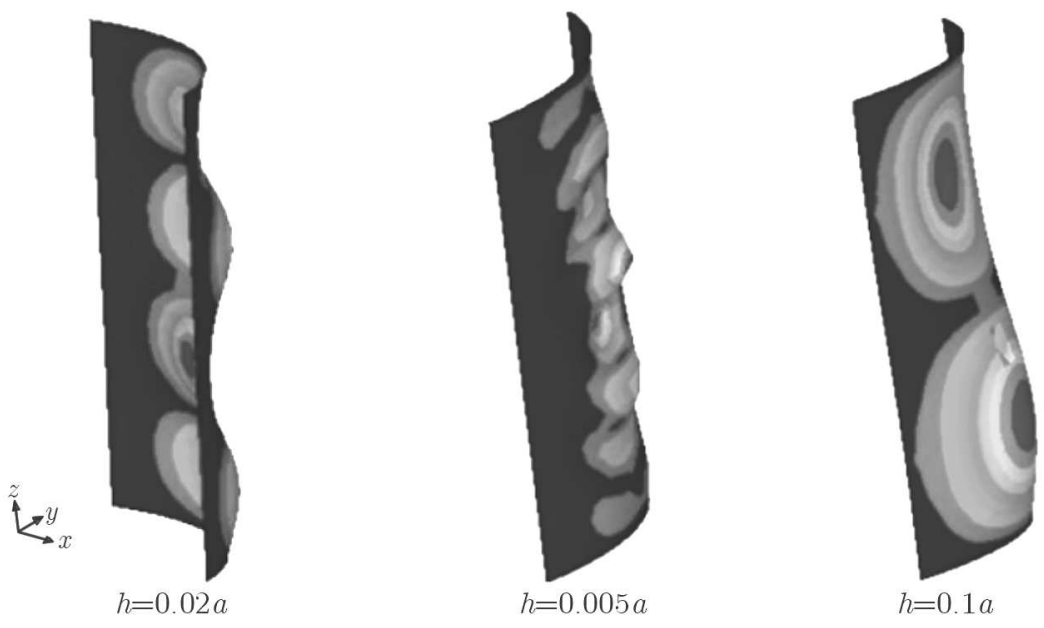

Fig. 7. Buckling mode shapes of the inhomogeneous $(K=1)$ panel (case 1$)$

It can be observed in Fig. 7 that when the thermal loading has a linear variation, the buckling temperature rises significantly. Then, the critical buckling temperatures with respect to the panel are plotted for the cylindrical panel with different temperatures at the outer surface under the action of non-uniform temperature rise in the radial direction. Assuming that the panel has 
$L=a=1 \mathrm{~m}, h=0.01 \mathrm{~m}$ and $\beta=1 \mathrm{rad}$, it is found that the variation of the outer surface temperature has a significant effect on the buckling temperature of the panel.
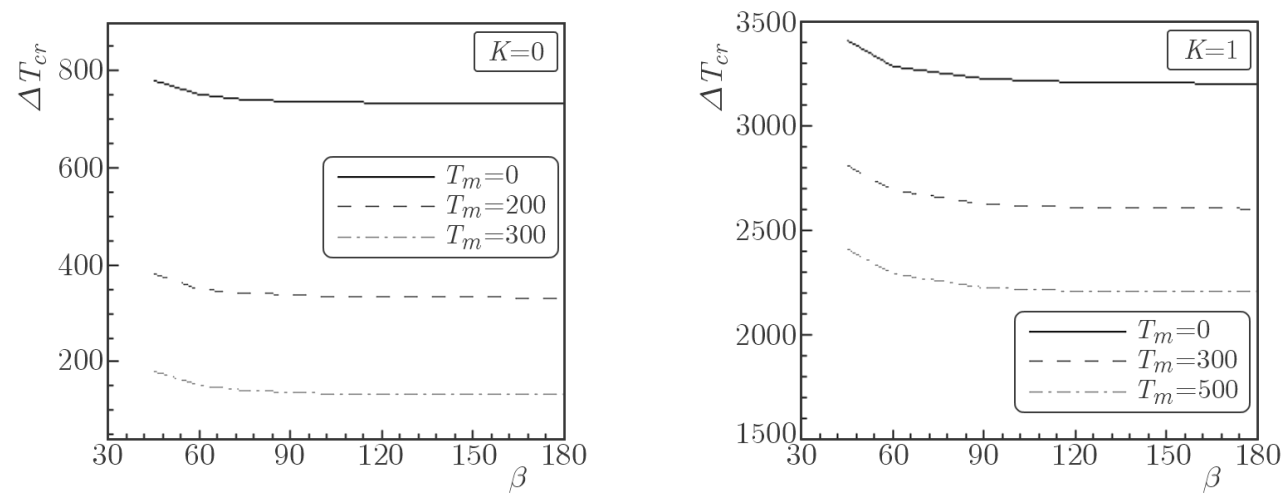

Fig. 8. Buckling temperature of the panel (case 1) versus $\beta$ for various values of $T_{m}$

Figure 9 demonstrates variation of the buckling temperature change versus aspect ratio $h / a$ of the panel (case 2). The results obtained through the present method are compared with the results obtained through the given governing equations in Breivik (1997). It should be noted that the buckling equations presented by Breivik (1997) are only solved for the shell (case 1), and here we resolve it for the panel (case 2) using equation (4.1).

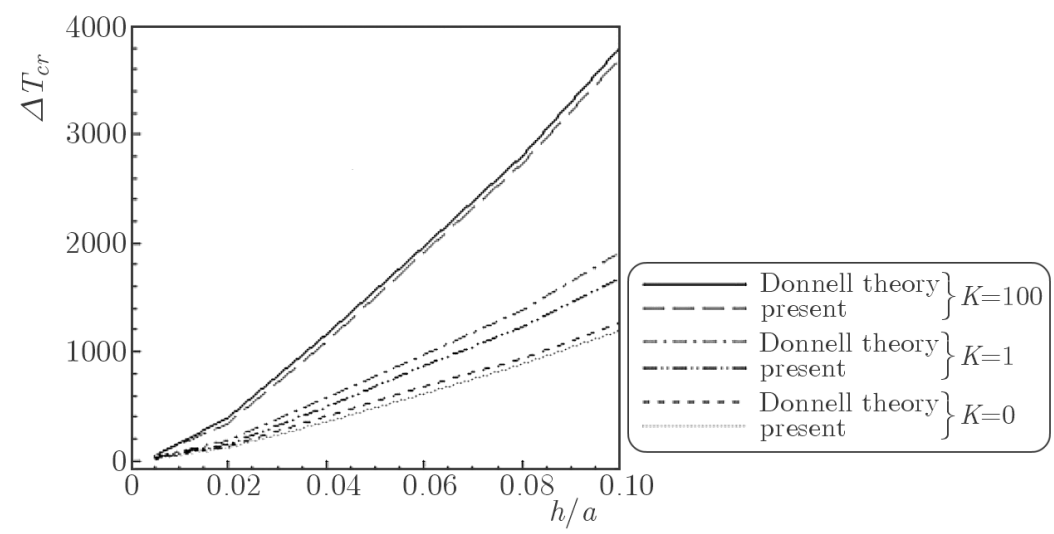

Fig. 9. Comparisons of the critical temperature of the panel (case 2) with different gradient indices under uniform temperature rise loading, $L=a=1 \mathrm{~m}, \beta=1 \mathrm{rad}\left(T_{m}=0\right)$

It is obvious that the results of the presented numerical method are in good agreement with the results issuing from Donnell's shell theory. As concluded above, the difference between the present results and those obtained based on Donnell's theory increase as the thickness of the panel grows. Buckling temperature changes against the thickness ratio for the panel (case 2) under the action of various loading conditions are illustrated in Figs. 10 and 11.

It can be found from Figs. 10 and 11 that the buckling temperature rises linearly when thickness of the panel increases. It is also clear that the critical buckling temperatures increase as the volume fractions of the ceramic increase. To study the effects of thermal loading variation in several directions and buckling temperature rises for the panel (case 2) with $L=a=1 \mathrm{~m}$, $\beta=0.8 \mathrm{rad}, h=0.005 \mathrm{~m}$ versus the index $K$ under combined temperature loadings are given in Table 1. 


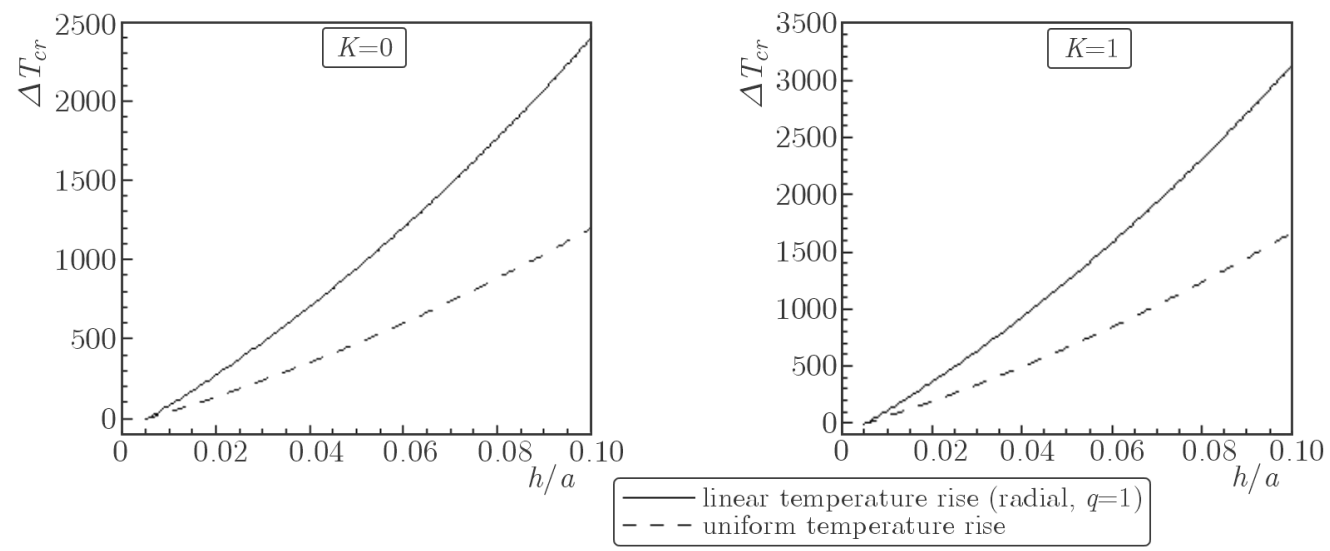

Fig. 10. Buckling temperature of panel (case 2) versus $h / a$ for uniform temperature rise and non-uniform loading in the radial direction, $\beta=0.8 \mathrm{rad}, L / a=1$

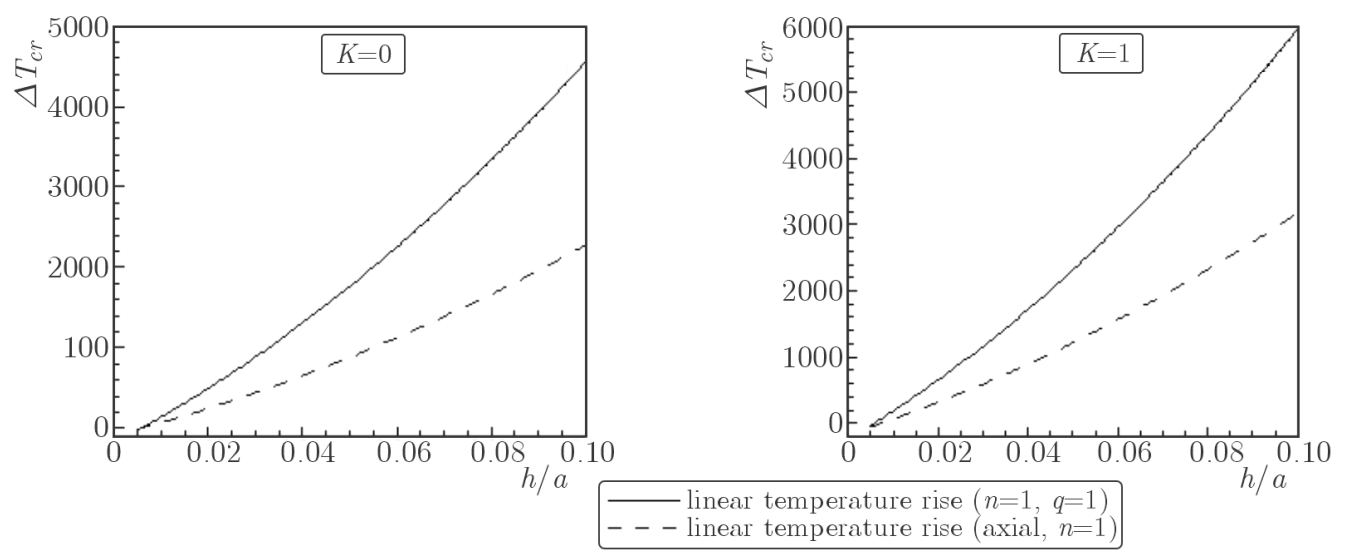

Fig. 11. Buckling temperature of the panel (case 2) versus $h / a$ for non-uniform temperature rise in the axial and radial direction, $\beta=0.8 \mathrm{rad}, L / a=1$

Table 1. Buckling temperature of the panel (case 2) versus $K$ for combined load conditions

\begin{tabular}{|c|c|c|c|c|c|c|}
\hline \multirow{2}{*}{$K$} & \multicolumn{2}{|c|}{$n=0$} & \multicolumn{2}{c|}{$n=0.5$} & \multicolumn{2}{c|}{$n=1$} \\
\cline { 2 - 7 } & $q=0$ & $q=1$ & $q=0$ & $q=1$ & $q=0$ & $q=1$ \\
\hline \hline 0 & 13.8 & 27.6 & 19 & 38 & 24.9 & 49.8 \\
\hline 1 & 18.7 & 35.1 & 26 & 48.9 & 34.1 & 64 \\
\hline 2 & 14.2 & 47 & 33.4 & 65.8 & 43.7 & 86 \\
\hline 5 & 31.9 & 65.6 & 44.3 & 89.8 & 57.6 & 118.8 \\
\hline 10 & 37.7 & 77.3 & 52.9 & 107.5 & 68.7 & 140.1 \\
\hline 20 & 41.5 & 84 & 57.8 & 117.3 & 75 & 152.5 \\
\hline 50 & 42.9 & 86.5 & 59.4 & 119.7 & 77.3 & 156.1 \\
\hline 100 & 43.2 & 87.6 & 59.8 & 119.8 & 77.6 & 156.8 \\
\hline
\end{tabular}

\section{Conclusion}

In this paper, buckling analysis of FG cylindrical panels under the action of thermal loading is carried out. Material properties are assumed to be temperature-independent and graded through the simple power law distribution in terms of the volume fractions of the constituents. The Differential Quadrature Method is used to discretize and solve buckling equations. The buckling analysis of such panels under the action of three types of thermal loadings, i.e. uniform temperature rise and non-uniform temperature rise in the axial and radial direction considering two 
types of boundary conditions, is carried out. From the present study, the following conclusions are drawn:

- Determination the critical loads by the use of equations extracted from Donnell's theory cause an overestimation when thickness of the panel increases. The results obtained by the three-dimensional buckling equations, as presented in this work, are more accurate in comparison with the results based on the Donnell shell theory.

- The critical buckling temperature $T_{c r}$ increases linearly with an increase in the thickness to mid-surface radius ratio $h / a$.

- For functionally graded cylindrical panels under various thermal loads, an increase in the volume fraction of the ceramic constituent increases the critical load.

\section{References}

1. Akbari Alashti R., Ahmadi S.A., 2014, Buckling of imperfect thick cylindrical shells and curved panels with different boundary conditions under external pressure, Journal of Theoretical and Applied Mechanics, 52, 1, 25-36

2. Alibeigloo A., Kani A.M., 2010, 3D free vibration analysis of laminated cylindrical shell integrated piezoelectric layers using differential quadrature method, Applied Mathematical Modeling, 34, 4123-4137

3. Bellman R.E., Casti J., 1971, Differential quadrature and long term integration, Journal of Mathematical Analysis and Applications, 34, 1, 235-238

4. BreiviK N.L., 1997, Thermal and mechanical response of curved composite panels, Dissertation submitted to the Faculty of the Virginia Polytechnic Institute and State University in partial fulfillment of the requirements for the degree of doctor of Philosophy in engineering mechanics

5. Chang J.S., Chui W.C., 1991, Thermal buckling analysis of antisymmetric laminated cylindrical shell panels, International Journal of Solids and Structures, 27, 1, 1295-1309

6. Haftchenari H., Darvizeh M., Darvizeh A., Ansari R., Sharma C.B., 2007, Dynamic analysis of composite cylindrical shells using Differential Quadrature Method (DQM), Composite Structures, 78, 292-298

7. JANG S.K., Bert C.W., Striz A.G., 1989, Application of differential quadrature to static analysis of structure components, International Journal for Numerical Methods in Engineering, 28, 261-577

8. Javaheri R., Eslami M.R., 2002a, Thermal buckling of functionally graded plates based on higher order theory, Journal of Thermal Stresses, 25, 603-625

9. JaVAheri R., Eslami M.R., 2002b, Thermal buckling of functionally graded plates, AIAA Journal, 40, 1, 162-169

10. Koizumi M., 1993, The concept of FGM, Ceramic Transactions, Functionally Gradient Materials, 34, 3-10

11. Mahayni M.A., 1966, Thermal buckling of shallow shells, International Journal of Solids and Structures, 2, 167-180

12. Mirfakhraei P., And Redekop D., 1998, Buckling of circular cylindrical shells by the differential quadrature method, International Journal of Pressure Vessels and Piping, 75, 347-353

13. Murphy K.D., Ferreira D., 2001, Thermal buckling of rectangular plates, International Journal of Solids and Structures, 38, 22/23, 3979-3994

14. Shahsiah R., Eslami M.R., 2003, Thermal buckling of functionally graded cylindrical shell, Journal of Thermal Stresses, 26, 277-294

15. SHu C., 2000, Differential Quadrature and its Application in Engineering, Springer-Verlag, London, UK 
16. Thornton E.A., 1993, Thermal buckling of plates and shells, Applied Mechanics Review, 46, 10, 485-506

17. Wu L.H., 2004, Thermal buckling of a simply supported moderately thick rectangular FGM plate, Composite Structures, 64, 2, 211-218

18. Wu L., JiAng Z., Liu J., 2005, Thermoelastic stability of functionally graded cylindrical shells, Composite Structures, 70, 60-68

19. Zhaо X., LiEw K.M., 2010, A mesh-free method for analysis of the thermal and mechanical buckling of functionally graded cylindrical shell panels, Computational Mechanics, 45, 297-310

20. Zhao X., YAng Y., Liew K.M., 2007, Geometrically nonlinear analysis of cylindrical shells using the element-free kp-Ritz method, Engineering Analysis with Boundary Elements, 31, 783-792

Manuscript received May 13, 2014; accepted for print July 15, 2015 